\title{
EDITORIAL
}

\section{Free Color Figures Online}

Powder Diffraction is pleased to announce a new feature for authors. Color figures will appear in the online journal free of charge if authors supply usable color graphics files in time for the production process. Manuscript figures will continue to appear in black and white in the print version with a notation "(Color online)" in the caption to alert readers that color is available online. Powder Diffraction online subscribers and those who buy individual articles online will be able to view figures in color electronically.

It will be the author's responsibility to provide a version of the figure in electronic form so that it appears clearly in color online and in black and white for print. It will also be the author's responsibility to note that a figure is "(Color online)" in its caption. More detailed information about figure submissions will be provided to authors of accepted manuscripts and outlined in the Notes for Authors.

Authors of Powder Diffraction can now communicate their science more effectively and likewise readers will better understand new data and information. 